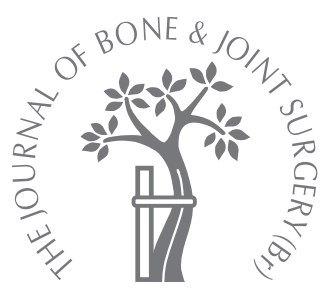

B. I. Eriksson,

A. K. Kakkar,

A. G. G. Turpie,

M. Gent,

T.-J. Bandel,

M. Homering,

F. Misselwitz,

M. R. Lassen

From the University

of Gothenburg,

Gothenburg, Sweden

B. I. Eriksson, $\mathrm{MD}, \mathrm{PhD}$

Orthopaedic Surgeon

Sahlgrenska University

Hospital/Östra, SE-41685

Gothenburg, Sweden.

A. K. Kakkar, MD, PhD,

Professor

Barts and the London School of

Medicine and Dentistry, Turner

Street, London E1 2AD, UK.

A. G. G. Turpie, MD,

Professor

McMaster University, 237

Barton Street East, Hamilton,

Ontario L8L 2X2, Canada.

M. Gent, DSc, Professor

Emeritus

McMaster University, 1200

Main Street West, Hamilton

Ontario L8N 3Z5, Canada.

- T.-J. Bandel, MD, Clinical

Development

M. Homering, $\mathrm{MSPH}$

Biostatistician

a. Misselwitz, $\mathrm{MD}, \mathrm{PhD}$

Clinical Development

Bayer Schering Pharma AG,

Aprather Weg 42096

Wuppertal, Germany.

M. R. Lassen, MD,

Orthopaedic Surgeon

Hørsholm Hospital, Usserød

Kongevej 102, DK-2970

Hørsholm, Denmark.

Correspondence should be sent to Dr B. I. Eriksson; e-mail: b.eriksson@orthop.gu.se

(C)2009 British Editorial Society of Bone and Joint Surgery doi:10.1302/0301-620X.91B5. $21691 \$ 2.00$

$J$ Bone Joint Surg $[\mathrm{Br}]$

2009;91-B:636-44.

Received 27 August 2008,

Accepted after revision 12

February 2009

\title{
Oral rivaroxaban for the prevention of symptomatic venous thromboembolism after elective hip and knee replacement
}

\author{
A once-daily dose of rivaroxaban $10 \mathrm{mg}$, an oral, direct Factor Xa inhibitor, was compared \\ with enoxaparin $\mathbf{4 0} \mathbf{~ m g}$ subcutaneously once daily for prevention of venous \\ thromboembolism in three studies of patients undergoing elective hip and knee \\ replacement (RECORD programme). \\ A pooled analysis of data from these studies $(n=9581)$ showed that rivaroxaban was \\ more effective than enoxaparin in reducing the incidence of the composite of symptomatic \\ venous thromboembolism and all-cause mortality at two weeks $(0.4 \%$ vs $0.8 \%$, respectively, \\ odds ratio $0.44 ; 95 \%$ confidence interval 0.23 to $0.79 ; p=0.005)$, and at the end of the \\ planned medication period $(0.5 \%$ vs $1.3 \%$, respectively; odds ratio $0.38 ; 95 \%$ confidence \\ interval 0.22 to $0.62 ; p<0.001)$. The rate of major bleeding was similar at two weeks $(0.2 \%$ \\ for both) and at the end of the planned medication period ( $0.3 \%$ vs $0.2 \%)$. \\ Rivaroxaban started six to eight hours after surgery was more effective than enoxaparin \\ started the previous evening in preventing symptomatic venous thromboembolism and all- \\ cause mortality, without increasing major bleeding.
}

Orthopaedic surgery may be associated with serious post-operative complications, such as venous thromboembolism in the form of deep-vein thrombosis (DVT) and/or pulmonary embolism, which can be fatal. It is estimated that $40 \%$ to $60 \%$ of patients undergoing major orthopaedic surgery of the hip or knee who do not receive thromboprophylaxis will develop DVT, as detected by venography. ${ }^{1}$ Up to one-third of these thrombi involve the proximal veins and are more likely to embolise than are distal venous thrombi, potentially resulting in a fatal pulmonary embolism. ${ }^{1}$ Despite such a high rate of venous thromboembolism in patients undergoing orthopaedic surgery, surgeons rarely witness them because they are often asymptomatic., ${ }^{2,3}$ Furthermore, because the first sign of venous thromboembolism could be a fatal pulmonary embolism, its incidence is thought to be underestimated owing to a lack of routine post mortem examinations. ${ }^{4}$

Low molecular weight heparins (LMWHs) are the standard of care for chemoprophylaxis of venous thromboembolism after major orthopaedic surgery in Europe. ${ }^{1}$ However, only $67 \%$ of physicians outside the United States use LMWHs in accordance with evidence-based guidelines. ${ }^{5,6}$
Rivaroxaban (Xarelto; Bayer Schering Pharma AG, Wuppertal, Germany) is a novel, oral, direct Factor Xa (FXa) inhibitor, ${ }^{7}$ which has been investigated as a $10 \mathrm{mg}$ once-daily dosage in the RECORD programme (REgulation of Coagulation in major Orthopaedic surgery Reducing risk of DVT and pulmonary embolism). The RECORD programme comprised four phase III studies, three of which (RECORD1 to 3) compared rivaroxaban $10 \mathrm{mg}$ once daily given post-operatively with enoxaparin $40 \mathrm{mg}$ once daily initiated the evening before surgery, in an investigation including more than 9500 patients (Fig. 1). The results of RECORD1, 2 and 3 have demonstrated the benefit of extended thromboprophylaxis after total hip replacement (THR; RECORD2), and showed that rivaroxaban $10 \mathrm{mg}$ once daily was significantly more effective than current standard chemothromboprophylaxis in preventing venous thromboembolism following THR and total knee replacement (TKR), with similar rates of bleeding (RECORD1 and 3, respectively). ${ }^{8-10}$

In this paper, we report the results of a pooled analysis of data from the three pivotal studies (RECORD1, 2 and 3), investigating whether rivaroxaban $10 \mathrm{mg}$ was more effective than the once-daily regimen of enoxaparin $40 \mathrm{mg}$ in reducing the incidence of the composite of symptomatic venous thromboembolism 


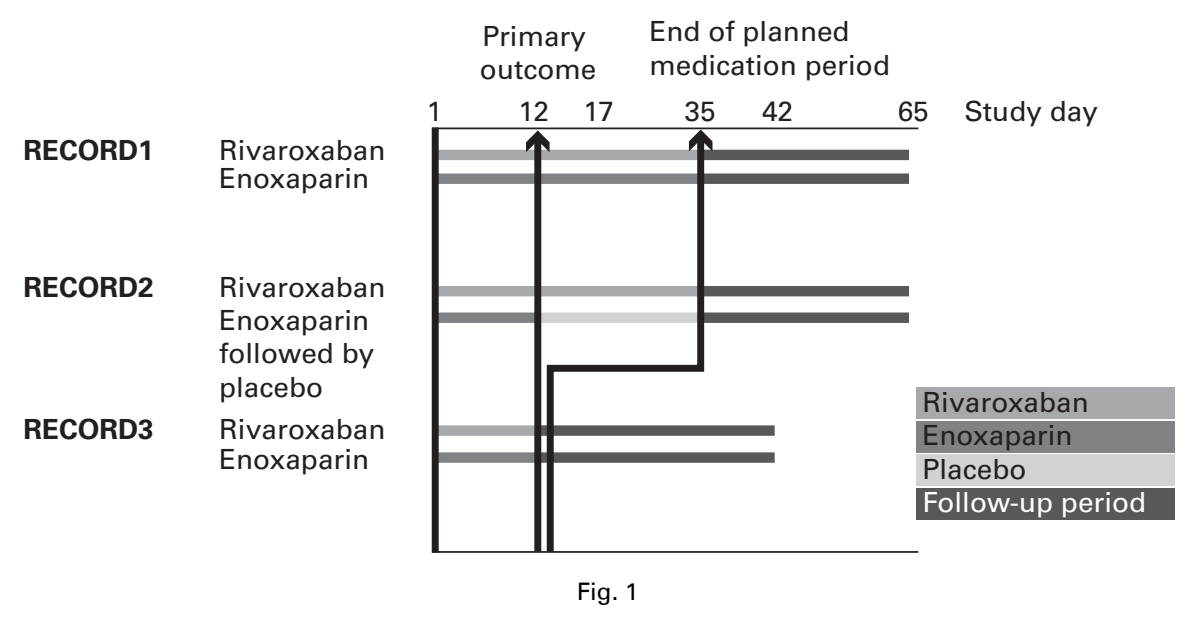

Chart showing the definition of outcomes in the pooled analysis of data from the RECORD1, 2 and 3 studies.

and all-cause mortality at two weeks (primary efficacy outcome) and at the end of the planned medication period (secondary efficacy outcome).

\section{Patients and Methods}

Patients aged 18 years or over scheduled to undergo a TKR or THR were eligible to be included in the study. Exclusion criteria were active bleeding, a high risk of bleeding contraindicating the use of LMWHs, any contraindication or condition precluding the use of enoxaparin or necessitating its dose adjustment, pregnancy or women who were breastfeeding. Other exclusion criteria included conditions prohibiting the use of bilateral venography; significant liver disease, such as acute clinical hepatitis, chronic active hepatatis and cirrhosis; severe renal impairment with a creatinine clearance of $<30 \mathrm{ml} / \mathrm{min}$; concomitant use of human immunodeficiency virus (HIV) protease inhibitors or fibrinolytic agents; planned intermittent pneumatic compression; or a requirement for anticoagulant therapy that could not be stopped.

Study design and medication. All the patients were randomised prior to surgery to receive a double-blind oral dose of rivaroxaban $10 \mathrm{mg}$ once daily or a subcutaneous dose of enoxaparin sodium $40 \mathrm{mg}$ once daily (Clexane/ Lovenox, Sanofi-Aventis, Frankfurt am Main, Germany). Enoxaparin was commenced 12 hours prior to surgery and then given six to eight hours following wound closure; rivaroxaban on the other hand was commenced six to eight hours following wound closure. Thereafter, study medications were administered every 24 hours in a double-dummy fashion: all patients received either placebo injections starting the evening prior to surgery or placebo tablets starting six to eight hours following wound closure.

The RECORD1 and RECORD2 studies were undertaken in patients undergoing THR. RECORD2 compared extended prophylaxis using rivaroxaban with short-term prophylaxis using enoxaparin; RECORD1 compared longterm prophylaxis using rivaroxaban and enoxaparin. RECORD3 compared rivaroxaban and enoxaparin for thromboprophylaxis following TKR.

The day of surgery was defined as day 1 . Patients underwent mandatory bilateral venography the day after the last dose of the study drug, at $36 \pm 6$ days in RECORD1 and 2, and at $13 \pm 2$ days in RECORD3. No further study medication was given after venography, although further thromboprophylaxis was continued at the investigator's discretion. Patients had a follow-up visit 30 to 35 days after the last dose of the study drug.

The studies were conducted in accordance with the International Conference on Harmonisation and Good Clinical Practice, ${ }^{11}$ and in the spirit of the Declaration of Helsinki ${ }^{12}$ and local regulations. Protocols were approved by the local ethics committee or institutional review board of each centre, and written, informed consent was obtained from all the patients before randomisation. All outcomes were assessed by the same central, independent adjudication committees blinded to the allocation of the study medication.

Deep-vein thrombosis was assessed on day 30 to 42 in RECORD1 and 2, and on day $13 \pm 2$ in RECORD3, or earlier if symptomatic, by ascending bilateral venography using the Rabinov and Paulin technique. ${ }^{13}$ All suspected DVTs had to be confirmed by venography (positive ultrasound had to be confirmed). In cases of suspected pulmonary embolism, pulmonary angiography, perfusion/ventilation lung scintigraphy with chest radiography or spiral CT were performed. If the patient died, all relevant documentation was collected, including autopsy reports if available.

The pre-specified primary efficacy outcome of this pooled analysis was the composite of symptomatic venous thromboembolism (i.e., symptomatic DVT and symptomatic non-fatal pulmonary embolism) and all-cause mortality up to two weeks, which included the enoxaparincontrolled period across all three studies, to allow for an 


\begin{tabular}{|c|c|c|}
\hline & $\begin{array}{l}\text { Rivaroxaban regimens } \\
(n=4657)\end{array}$ & $\begin{array}{l}\text { Enoxaparin regimens } \\
(n=4692)\end{array}$ \\
\hline Female (\%) & $2744 \quad(58.9)$ & $2714(57.8)$ \\
\hline Mean age in yrs (range) & 63.8 (18 to 93$)$ & 64.0 (18 to 93$)$ \\
\hline Mean weight in kg (range) & 77.6 (37.0 to 158.8$)$ & $78.2(33.2$ to 157.0$)$ \\
\hline Mean body mass index in $\mathrm{kg} / \mathrm{m}^{2}$ (range) & $28.0(15.6$ to 54.7$)$ & $28.2(15.2$ to 59.0$)$ \\
\hline \multicolumn{3}{|l|}{ Race (\%) } \\
\hline Caucasian & $3840(82.5)$ & $3844(81.9)$ \\
\hline History of venous thromboembolism (\%) & $105(2.3)$ & $117(2.5)$ \\
\hline Total hip replacement (patients) & $\mathrm{n}=3437$ & $\mathrm{n}=3453$ \\
\hline \multicolumn{3}{|l|}{ Type of surgery (\%) } \\
\hline Bilateral & $28(0.8)$ & $38 \quad(1.1)$ \\
\hline Primary & $3287(95.6)$ & $3275(94.8)$ \\
\hline Revision & $118(3.4)$ & $136 \quad(3.9)$ \\
\hline Details missing & $32(0.9)$ & $40 \quad(1.2)$ \\
\hline Cemented fixation & $1478(43.0)$ & $1477(42.8)$ \\
\hline Mean duration of surgery in mins (range) & $97(27$ to 480$)$ & 98 (25 to 595$)$ \\
\hline \multicolumn{3}{|l|}{ Type of anaesthesia (\%) } \\
\hline General only & $1002(29.2)$ & $981 \quad(28.4)$ \\
\hline General and regional & $300(8.7)$ & $319 \quad(9.2)$ \\
\hline Regional only & $2102(61.2)$ & $2113(61.2)$ \\
\hline None/missing & $33(1.0)$ & $40(1.2)$ \\
\hline Total knee replacement (patients) & $\mathrm{n}=1220$ & $\mathrm{n}=1239$ \\
\hline \multicolumn{3}{|l|}{ Type of surgery (\%) } \\
\hline Bilateral & $20 \quad(1.6)$ & $29(2.3)$ \\
\hline Primary & $1176 \quad(96.4)$ & $1186(95.7)$ \\
\hline Revision & $24 \quad(2.0)$ & $30(2.4)$ \\
\hline None/missing & $20 \quad(1.6)$ & $23(1.9)$ \\
\hline Mean duration of surgery in mins (range) & $96.4(26$ to 500$)$ & 97.1 (28 to 315$)$ \\
\hline \multicolumn{3}{|l|}{ Type of anaesthesia (\%) } \\
\hline General only & $227(18.6)$ & $242(19.5)$ \\
\hline General and regional & $188(15.4)$ & $201(16.2)$ \\
\hline Regional only & $786(64.4)$ & $774(62.5)$ \\
\hline None/missing & $19(1.6)$ & $22(1.8)$ \\
\hline
\end{tabular}

unbiased comparison with enoxaparin (Fig. 1). The secondary exploratory efficacy outcome was the same combination of events until the end of the planned medication period, which was five weeks (up to day 42) in RECORD1 and RECORD2, and included the placebo-controlled period in RECORD2, and two weeks (up to day 17) in RECORD3 (Fig. 1).

The main safety outcomes were the incidence of major bleeding events up to two weeks post-operatively, again representing the enoxaparin-controlled period across all three studies, and until the end of the planned medication period. Major bleeding was defined as bleeding that was fatal, into a critical site such as the retroperitoneal space, intracranially, intraocularly, or intraspinally, required re-operation, or clin- ically overt extra-surgical site bleeding associated with a fall in haemoglobin of $\geq 2 \mathrm{~g} / \mathrm{dl}$ or requiring infusion of 2 or more units of whole blood or packed cells. Other safety outcomes included any on-treatment bleeding up to two days after the last intake of study medication, excessive wound haematoma, surgical-site bleeding, adverse event, and all-cause mortality over the entire study period comprising the planned medication period plus 30-day follow-up.

Laboratory variables including liver function tests were monitored during the studies and at follow-up.

Statistical analysis and appropriateness of data pooling. The appropriateness of pooling the efficacy data across the three studies was determined by testing the homogeneity of the study odds ratios using the modified Breslow-Day test. 
Table II. Incidence of the composite of symptomatic venous thromboembolism (VTE) and all-cause mortality up to two weeks (prespecified primary outcome of pooled analysis) and during the planned medication period (secondary outcome of pooled analysis) in patients receiving rivaroxaban $10 \mathrm{mg}$ once daily and enoxaparin $40 \mathrm{mg}$ in the RECORD1, 2 and 3 studies ${ }^{*}$ (safety population, $\mathrm{n}=9349$ )

\begin{tabular}{|c|c|c|c|c|c|}
\hline & $\begin{array}{l}\text { Rivaroxaban regimen } \\
\text { (10 mg once daily) } \\
\text { number }(\%)\end{array}$ & $\begin{array}{l}\text { Enoxaparin regimen } \\
\text { ( } 40 \mathrm{mg} \text { once daily) } \\
\text { number }(\%)\end{array}$ & $\begin{array}{l}\text { Odds ratio }{ }^{\dagger} \text { vs enoxaparin } \\
(95 \% \mathrm{Cl})\end{array}$ & p-value for difference & p-value for homogeneity ${ }^{\ddagger}$ \\
\hline \multicolumn{6}{|c|}{ Symptomatic VTE and all-cause mortality up to two weeks } \\
\hline RECORD1 & $7(0.3)$ & $8(0.4)$ & $0.88(0.27$ to 2.78$)$ & - & - \\
\hline RECORD2 & $2(0.2)$ & $5(0.4)$ & $0.40(0.04$ to 2.45$)$ & - & - \\
\hline RECORD3 & $8(0.7)$ & $26(2.1)$ & $0.31(0.12$ to 0.70$)$ & - & - \\
\hline Pooled data ${ }^{*}$ & $17(0.4)$ & $39(0.8)$ & $0.44(0.23$ to 0.79$)$ & 0.005 & 0.264 \\
\hline \multicolumn{6}{|c|}{ Symptomatic VTE and all-cause mortality during the planned medication period } \\
\hline RECORD1 & $10(0.5)$ & $15(0.7)$ & $0.67(0.27$ to 1.60$)$ & - & - \\
\hline RECORD2 & $5(0.4)$ & $20(1.6)$ & 0.25 (0.07 to 0.68$)$ & - & - \\
\hline RECORD3 & $8(0.7)$ & $26(2.1)$ & $0.31(0.12$ to 0.70$)$ & - & - \\
\hline Pooled data ${ }^{*}$ & $23(0.5)$ & $61(1.3)$ & $0.38(0.22$ to 0.62$)$ & $<0.001$ & 0.224 \\
\hline
\end{tabular}

The primary efficacy and safety outcome analyses were pre-specified before the results of the first RECORD study were unblinded. Pre-specification included events that occurred up to two weeks post-operatively in the extended prophylaxis studies RECORD1 and 2. For RECORD2, analyses up to two weeks considered events that occurred until two days after the last enoxaparin injection/matching placebo injection. For RECORD1, analyses up to two weeks considered bleeding events only up to day 16; all other timings for counting events were analogous to RECORD3. On-treatment adverse events, including bleeding, were defined as occurring after the first dose of study medication and no more than two days after the last dose was administered.

All outcomes were analysed in all patients who had received at least one dose of study medication, including placebo injection (i.e. the safety population), because symptomatic events were detected continuously throughout the studies and were not dependent on adequate venography. The primary efficacy outcome of each individual study was a combination of events, including asymptomatic DVT detected by venography.

Although the primary efficacy and main safety analyses were pre-specified, no $\alpha$-adjustments were made for the multiplicity of outcomes and comparisons (nominal p-values). Two-sided $95 \%$ confidence intervals (CI) were used to quantify the random error associated with the data.

Comparisons of efficacy outcomes between the two groups were based on odds ratios (OR). Exact methods for a studystratified pooled estimate were obtained with a series of three $2 \times 2$ tables and two-sided $95 \%$ CIs were applied. The twosided pooled p-values correspond to the exact $95 \%$ CI.

All safety data were analysed descriptively with twosided p-values based on Fisher's exact test for single $2 \times 2$ tables. Statistical significance was set at a p-value $<0.05$.

\section{Results}

A total of 9581 patients were randomised across 487 centres in 38 countries between February 2006 and June 2007. Similar numbers of patients were randomised to receive rivaroxaban $(n=4772)$ and enoxaparin $(n=4809)$, and a similar number were excluded from the safety population because they did not receive any study medication (115 and 117 in the rivaroxaban and enoxaparin groups, respectively). Therefore, the safety population, which was used for all analyses, comprised a total of 9349 patients, of whom 4657 were in the rivaroxaban group and 4692 in the enoxaparin group. The two groups were well balanced in terms of baseline and surgical characteristics (Table I).

Similar numbers of patients withdrew prematurely from the studies in each group (rivaroxaban: 518 (10.9\%); enoxaparin: $584(12.1 \%)$ for various reasons, which included consent withdrawn (rivaroxaban: $240(5.0 \%)$, enoxaparin: $226(4.7 \%)$; protocol violation (rivaroxaban: $56(1.2 \%)$, enoxaparin: $70(1.5 \%)$; non-compliance with study medication (rivaroxaban: $31(0.6 \%)$, enoxaparin: $28(0.6 \%)$, adverse events (rivaroxaban: 162 (3.4\%), enoxaparin: 188 $(3.9 \%)$; and lost to follow-up (rivaroxaban: $5(0.1 \%)$; enoxaparin: $14(0.3 \%)$.

Efficacy outcomes. Rivaroxaban significantly reduced the incidence of the pre-specified primary outcome (the composite of symptomatic venous thromboembolism and all-cause mortality at two weeks) compared with enoxaparin $(0.4 \%$ and $0.8 \%$, respectively, OR $0.44,95 \%$ CI 0.23 to 0.79 ; $\mathrm{p}=0.005$; Table II). The rivaroxaban regimen also reduced the incidence of the secondary exploratory efficacy outcome (the composite of symptomatic venous thromboembolism and all-cause mortality at the end of the planned medication period) more effectively than the enoxaparin regimen $(0.5 \%$ and $1.3 \%$ respectively, OR $0.38,95 \%$ CI 0.22 to 0.62 ; $\mathrm{p}<0.001)$. A review of the occurrence of the composite of 


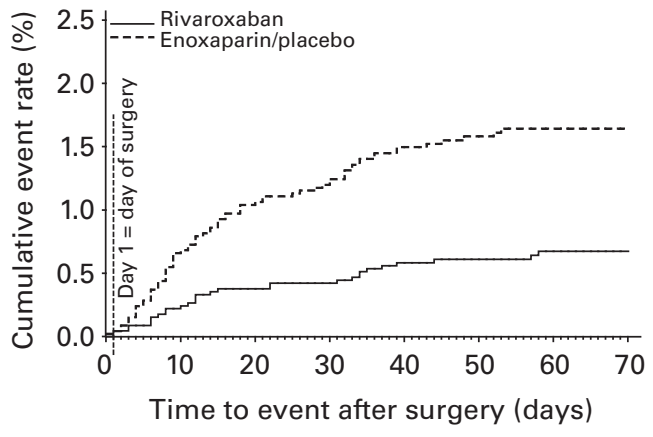

Number of patients at risk

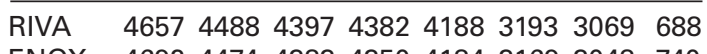
ENOX $4692447443834350418431693048 \quad 740$

Fig. 2

Kaplan-Meier survival curve showing cumulative incidence of symptomatic venous thromboembolism and all-cause mortality over the total study duration in patients (valid for safety population) receiving the rivaroxaban regimen and the enoxaparin regimen in the RECORD1- 3 studies. In RECORD1, patients received long-term thromboprophylaxis (five weeks) with rivaroxaban $10 \mathrm{mg}$ once daily or enoxaparin $40 \mathrm{mg}$ once daily; in RECORD2, patients received rivaroxaban $10 \mathrm{mg}$ once daily for five weeks or enoxaparin $40 \mathrm{mg}$ once daily for two weeks followed by placebo and in RECORD3, patients received rivaroxaban $10 \mathrm{mg}$ once daily or enoxaparin $40 \mathrm{mg}$ once daily for two weeks. All symptomatic venous thromboembolism events and deaths reported in the studies.

symptomatic venous thromboembolism and all-cause mortality over time (Fig. 2) shows an early and sustained separation of the curves in favour of rivaroxaban, with the curves continuing to separate over time during the treatment phase, and treatment differences persisting at the end of the study.

Exploratory analysis showed a reduction in the incidence of the composite of all-cause mortality and pulmonary embolism with the rivaroxaban regimens compared with the enoxaparin regimens during the entire study period $(0.3 \%$ and $0.7 \%$, respectively, OR $0.52,95 \%$ CI 0.26 to 0.98 : $\mathrm{p}=0.042$ ). The results for the individual components, pulmonary embolism and all-cause mortality, showed a similar pattern, with both components contributing to the overall effect. Safety outcomes. The incidence of major bleeding events was similar between the rivaroxaban and enoxaparin regimens, irrespective of the duration of treatment (Table III).

There were a total of five bleeding events into a critical organ until the end of the planned medication period (rivaroxaban: two $(<0.1 \%)$, enoxaparin: three $(0.1 \%))$. In the rivaroxaban group, one event was adjudicated as a haemorrhagic puncture related to spinal anaesthesia, but this occurred after the injection of placebo the evening before surgery and prior to the first intake of rivaroxaban. This patient experienced no neurological signs or symptoms of compression. The other event was in a patient with Gaucher's disease and a history of intraocular bleeding who experienced another episode of intraocular bleeding. All three events in the enoxaparin group were adjudicated as intraspinal bleeding/haemorrhagic puncture: two episodes of spinal bleeding (one was an extradural haematoma leading to prolonged hospitalisation and permanent drug discontinuation, but with eventual resolution of the symptoms; the other was a spinal catheter-related complication involving major bleeding leading to drug discontinuation, although the drug was restarted after the bleeding had resolved) and one haemorrhagic puncture related to spinal anaesthesia (classified as clinically overt bleeding) which led to drug discontinuation.

There was a similar incidence of the other bleeding events that comprised the major bleeding category, between the two groups: events leading to re-operation (rivaroxaban: six $(0.1 \%)$, enoxaparin: five $(0.1 \%)$; clinically overt extra-surgical site bleedings associated with either $\mathrm{a} \geq 2 \mathrm{~g} / \mathrm{dl}$ reduction in haemoglobin or transfusion of 2 or more units of whole blood or packed cells (rivaroxaban: four $(0.1 \%)$, enoxaparin: one $(<0.1 \%))$. One fatal bleeding event occurred during surgery in a patient randomised to receive rivaroxaban, who had received a placebo injection the evening before surgery but had not received any rivaroxaban at the time of complication. Although the patient had not received rivaroxaban, this event was classified as an ontreatment event, in accordance with the study protocol.

The incidences of adjudicated haemorrhagic wound complications, a composite of excessive wound haematoma and reported surgical-site bleeding, and wound infections were also similar between the two groups up to two weeks and at the end of the planned medication period (Table III).

The incidence of adverse events was also similar between the two regimens; the differences between the groups did not exceed $1 \%$ (Medical Dictionary for Regulatory Activities ${ }^{14}$ preferred level at term), except for investigatorreported DVT, which was lower with the rivaroxaban regimen (up to two weeks: $2.7 \%$ vs $4.4 \%$ and end of planned medication period: $4.2 \%$ vs $7.7 \%$ ). There was no evidence of compromised liver function attributable to rivaroxaban.

\section{Discussion}

The RECORD1, 2 and 3 studies compared rivaroxaban and enoxaparin for venous thromboembolism prevention after major orthopaedic surgery in 9581 patients. This pooled analysis of data from these studies demonstrates that rivaroxaban $10 \mathrm{mg}$ is superior to enoxaparin $40 \mathrm{mg}$ for the reduction of the composite of symptomatic venous thromboembolism and all-cause mortality at two weeks. This reduction, which occurred during the early enoxaparincontrolled two-week period $(\mathrm{p}=0.005)$, was sustained with the rivaroxaban regimen over the entire medication period $(\mathrm{p}<0.001)$. This is the first study to show such a comprehensive clinical benefit with a new oral anticoagulant involving a large number of patients.

We recognise that these studies are heterogeneous in terms of treatment duration. Therefore, the primary efficacy outcome was analysed at up to two weeks postoperatively, i.e., during the enoxaparin-controlled period, 
Table III. Safety outcomes* at two weeks, at the end of the planned medication period, and during the entire study period (planned medication period plus follow-up) in patients receiving rivaroxaban $10 \mathrm{mg}$ once daily or enoxaparin 40 mg once daily across the RECORD1 to 3 studies (safety population, $\mathrm{n}=9349$ )

\begin{tabular}{|c|c|c|c|}
\hline & $\begin{array}{l}\text { Rivaroxaban regimen ( } 10 \mathrm{mg} \text { once } \\
\text { daily) }(\%) \text { (number }=4657 \text { ) }\end{array}$ & $\begin{array}{l}\text { Enoxaparin regimen }(40 \mathrm{mg} \text { once } \\
\text { daily) }(\%)(\text { number }=4692)\end{array}$ & $\begin{array}{l}\text { p-value for } \\
\text { difference }\end{array}$ \\
\hline \multicolumn{4}{|l|}{ Up to 2 weeks } \\
\hline Any on-treatment bleeding & $249(5.3)$ & $242(5.2)$ & 0.711 \\
\hline On-treatment major bleeding & $11(0.2)$ & $9(0.2)$ & 0.662 \\
\hline Leading to re-operation & $6(0.1)$ & $5(0.1)$ & - \\
\hline On-treatment non-major bleeding & $239(5.1)$ & $233(5.0)$ & - \\
\hline On-treatment clinically relevant non-major bleeding & $120(2.6)$ & $109(2.3)$ & - \\
\hline Haemorrhagic wound complications ${ }^{\ddagger}$ & $75(1.6)$ & $80(1.7)$ & - \\
\hline Other on-treatment non-major bleeding & $127(2.7)$ & $133(2.8)$ & - \\
\hline Wound infections ${ }^{\S}$ & $52(1.1)$ & $49(1.0)$ & - \\
\hline Nausea $^{\S}$ & $503(10.8)$ & $509(10.8)$ & - \\
\hline Vomiting $^{\S}$ & $448(9.6)$ & $478(10.2)$ & - \\
\hline Leading to discontinuation of study drug & $3(<0.1)$ & $4(0.1)$ & - \\
\hline Death & $4(0.1)$ & $7(0.1)$ & \\
\hline \multicolumn{4}{|l|}{ End of planned medication period } \\
\hline Any on-treatment bleeding & $274(5.9)$ & $259(5.5)$ & 0.475 \\
\hline On-treatment major bleeding & $14(0.3)$ & $9(0.2)$ & 0.305 \\
\hline On-treatment non-major bleeding & $261(5.6)$ & $250(5.3)$ & - \\
\hline On-treatment clinically relevant non-major bleeding & $138(3.0)$ & $115(2.5)$ & - \\
\hline Haemorrhagic wound complications ${ }^{\ddagger}$ & $79(1.7)$ & $83(1.8)$ & - \\
\hline Other non-major bleeding & $136(2.9)$ & $144(3.1)$ & - \\
\hline Wound infections & $80(1.7)$ & $75(1.6)$ & - \\
\hline Patients receiving blood transfusions & $2314(49.7)$ & $2338(49.8)$ & - \\
\hline $\begin{array}{l}\text { Volume of blood transfused }-\mathrm{ml} \text { (median and } 10 \% \text { to } 90 \% \\
\text { percentile in patients who had blood transfusion }\end{array}$ & $600.0(250$ to 1230$)$ & $600.0(250$ to 1300$)$ & - \\
\hline Patients with a post-operative drain & $3667(78.7)$ & $3687(78.6)$ & - \\
\hline Volume in drain: $\mathrm{ml}$ (median and $10 \%$ to $90 \%$ percentile) & $535(180$ to 1120$)$ & $520(170$ to 1070$)$ & - \\
\hline Adverse events leading to discontinuation of study medication & $170(3.7)$ & $220(4.7)$ & - \\
\hline Nausea ${ }^{\S}$ & $517(11.1)$ & $519(11.1)$ & - \\
\hline Vomiting $^{\S}$ & $452(9.7)$ & $482(10.3)$ & - \\
\hline Leading to discontinuation of study drug & $3(<0.1)$ & $4(0.1)$ & - \\
\hline Death & $6(0.1)$ & $13(0.3)$ & - \\
\hline \multicolumn{4}{|l|}{ Entire study period } \\
\hline Cardiovascular events & $23(0.5)$ & $23(0.5)$ & - \\
\hline Myocardial infarction & $12(0.3)$ & $13(0.3)$ & - \\
\hline Ischaemic stroke & $8(0.2)$ & $5(0.1)$ & - \\
\hline Cardiovascular death & $5(0.1)$ & $4(0.1)$ & - \\
\hline Unexplained death & $0(0.0)$ & $2(<0.1)$ & - \\
\hline Non-fatal pulmonary embolism or death & $16(0.3)$ & $31(0.7)$ & 0.040 \\
\hline Non-fatal pulmonary embolism & $9(0.2)$ & $12(0.3)$ & - \\
\hline Fatal pulmonary embolism & $0(0.0)$ & $3(<0.1)$ & - \\
\hline Death (all causes) & $7(0.2)^{\pi}$ & $19(0.4)$ & 0.030 \\
\hline
\end{tabular}

* data are number (\%) of patients, except where indicated (patients may have had more than one event, or an event that falls into one or more categories)

$\dagger$ Fisher's exact two-sided p-value

‡ composite of excessive wound haematoma and reported surgical-site bleeding

$\S$ on-treatment adverse events that occurred up to two days after the last dose of study medication

If there was an additional death in the rivaroxaban group in a patient who did not receive any blinded study medication and did not undergo surgery (killed in a road traffic accident), and was therefore not included in the safety population 
across all three studies, which removes any possible effects of the different durations of rivaroxaban and enoxaparin and allows appropriate assessment of rivaroxaban versus the active comparator. Furthermore, we included a statistical assessment of homogeneity of the study-specific odds ratio estimates, which did not reveal evidence of heterogeneity, suggesting that it is appropriate to pool these data.

The secondary exploratory efficacy outcome consisted of the same combination of events as the primary efficacy outcome, i.e., the composite of symptomatic venous thromboembolism and all-cause mortality, but in this instance until the end of the planned medication period. Venous thromboembolic events can occur after the second post-operative week, so this secondary exploratory efficacy outcome analysis was necessary, even though it was conducted in patients receiving prophylaxis for different durations. For the RECORD1 and 2 studies, which were conducted in patients undergoing elective THR, the study period for this secondary efficacy outcome was five weeks (up to day 42), which was the end of the planned medication period. This is particularly appropriate, as recent findings from the Global Orthopaedic Registry show that the mean time to venous thromboembolism is 21.5 days (SD 22.5) for THR. ${ }^{15}$ For the RECORD3 study, which was conducted in patients undergoing elective TKR, the study period for the secondary efficacy outcome was two weeks (up to day 17). Again, this duration is appropriate, as the recent findings from the Global Orthopaedic Registry show that the mean time to venous thromboembolism is 9.7 days (SD 14.1). The Kaplan-Meier estimates of the occurrence of the composite of symptomatic venous thromboembolism and all-cause mortality over the total study duration (Fig. 2) show an early and sustained separation of the curves in favour of rivaroxaban, with the curves continuing to separate over time during the treatment phase and treatment differences persisting at the end of the study (including the 30 to 35 days of follow-up).

Finally, safety outcomes were measured both in the two-week period to have the most homogeneous group, and up to two days after the last intake of study medication (on-treatment). The incidence of major bleeding was similar with both rivaroxaban and enoxaparin regimens up to two weeks $(0.2 \%$ for both) and with both rivaroxaban and enoxaparin regimens at the end of the medication period $(0.3 \%$ for rivaroxaban and $0.2 \%$ for enoxaparin).

The same definition of major bleeding used across the three RECORD studies analysed here excluded woundrelated bleeding events, unless they led to a re-operation. An important difference between the RECORD programme and other contemporary studies on VTE prevention has been that surgical-site bleeding events were not included as part of clinically major bleeding. When different definitions of safety are used, it is difficult to compare safety findings between the RECORD programme and phase III studies performed on other drugs. However, when a pre-defined algorithm taking into account the clinically overt surgical-site bleeding events resulting in a fall in haemoglobin of $\geq 2 \mathrm{~g} / \mathrm{dl}$ and the transfusion of two or more units of blood or packed cells was applied, the resulting major bleeding event rates remained similar between both the study groups. At day 12, when the surgical-site bleeding events that meet the haemoglobin and transfusion criteria were included, $81(1.7 \%)$ of rivaroxaban patients and $68(1.4 \%)$ of enoxaparin patients experienced a major bleeding event based on these surrogate criteria. Comparing rivaroxaban and enoxaparin, it can be said that, irrespective of the outcomes used, the rate of bleeding events was similar between the groups.

Of the major bleeding events occurring into a critical organ until the end of the planned medication period, four out of five were adjudicated as intraspinal bleeding/haemorrhagic puncture. It is recognised that spinal/epidural (neuraxial) anaesthesia or spinal/epidural puncture, when used in patients treated with antithrombotic agents for the prevention of thromboembolic complications increase the risk for developing a spinal/epidural haematoma, ${ }^{16}$ which can result in long-term or permanent neurological impairment. In order to minimise a potential risk of bleeding, epidural catheter placement and removal should preferably be undertaken when the anticoagulant effect of the drug is low. ${ }^{17}$ An epidural catheter should not be removed earlier than 18 hours after the last administration of rivaroxaban, and the next dose should be administered no earlier than six hours after the removal of the catheter. ${ }^{18}$ Further, if traumatic puncture occurs, the administration of rivaroxaban should be delayed for 24 hours. ${ }^{18}$ Patients should be frequently monitored for signs and symptoms of neurological impairment. If neurological compromise is noted, urgent diagnosis (i.e. MRI) and treatment is necessary (spinal decompression).

Haemorrhagic wound complications which did not qualify as major bleeding events in these studies unless they led to re-operation, were included in the assessment of nonmajor bleeding and were similar between the two groups at either two weeks (rivaroxaban $1.6 \%$; enoxaparin $1.7 \%$ ) or the end of the planned medication period (rivaroxaban $1.7 \%$; enoxaparin $1.8 \%$ ). Likewise, the incidence of wound infections was similar across the two groups (two weeks: $1.1 \%$ and $1.0 \%$; end of planned medication period: $1.7 \%$ and $1.6 \%$, respectively).

The incidence of cardiovascular events (cardiovascular death, ischaemic stroke and myocardial infarction) during the entire study period was similar with both regimens (both $0.5 \%$ ), and there was no imbalance in the number of adjudicated, non-fatal or fatal cardiovascular events between the groups (Table III).

Post-operative vomiting can be caused by different factors, such as the use of certain anaesthetics, stress, trauma from surgery, or the peri-operative use of analgesics such as opioids. ${ }^{19}$ As rivaroxaban has a fast onset of action, with time to peak reached within 2.5 to four hours after administration, it might already have been absorbed prior to the onset of any vomiting. 
Finally, it is important to consider whether the superior efficacy demonstrated by rivaroxaban in reducing symptomatic venous thromboembolism and all-cause mortality up to two weeks was maintained throughout the study and the follow-up period. The reduction of all-cause mortality and pulmonary embolism at the end of the entire study period with the rivaroxaban regimen followed a similar pattern as the primary outcome.

A meta-analysis comparing LMWHs and vitamin $\mathrm{K}$ antagonists failed to demonstrate the superiority of LMWHs in terms of reduction of symptomatic venous thromboembolism and death. ${ }^{20}$ In a meta-analysis of four pivotal fondaparinux trials including 7344 patients, it was shown that symptomatic venous thromboembolism plus death occurred in $1 \%$ of patients, with no difference between fondaparinux and enoxaparin in clinical outcome. ${ }^{21}$ Furthermore, evidence-based guidelines recommend against the use of acetylsalicyclic acid for venous thromboembolism prevention, because its use only reduces the risk of DVT by $26 \%$ and $13 \%$ against $70 \%$ and $52 \%$ for LMWHs, in THR and TKR, respectively. ${ }^{1}$

There was one fatal bleeding event in a patient randomised to receive rivaroxaban, which happened during surgery and before the patient received the first dose of rivaroxaban. The rivaroxaban regimen was associated with fewer deaths from all causes as compared with the enoxaparin regimen (Table III), and with a lower incidence of non-fatal pulmonary embolism or death (16 vs 31, respectively) over the entire study period.

Although there is no specific antidote to reverse the effects of rivaroxaban, preclinical studies suggest that recombinant activated Factor VIIa (rFVIIa) or Factor VIII inhibitor bypass activity (FEIBA) may reverse the effects of high-dose rivaroxaban. ${ }^{22-24}$ If stategies such as delaying the next dose of rivaroxaban or discontinuation, mechanical compression, surgical intervention, and blood product transfusion fail to control bleeding, administration of rFVIIa or FEIBA may be considered, but there is currently no clinical experience of this strategy with rivaroxoban.

There are known limitations associated with the use of enoxaparin, such as the inconvenience of subcutaneous administration after the patient is discharged. In patients who underwent THR and TKR only $77 \%$ and $75 \%$, respectively who received LMWH in hospital continued to receive it after discharge. ${ }^{6}$ However, the most recent American College of Chest Physicians guidelines recommend the use of extended prophylaxis (up to 35 days) following both THR and TKR..$^{5}$ Therefore, an oral anticoagulant such as rivaroxaban, with superior efficacy and similar safety to enoxaparin $40 \mathrm{mg}$, would be useful in reducing the risk of venous thromboembolism following elective hip and knee replacements.

Symptomatic venous thromboembolic events are costly ${ }^{25}$ and have a negative effect on the function and mobility of patients. Therefore, the results of this pooled analysis have important economic implications, as they show that the use of rivaroxaban is associated with significant reductions in the incidence of such symptomatic events $(0.4 \%$ and $0.8 \%$, respectively, OR $0.44,95 \%$ CI 0.23 to $0.79 ; \mathrm{p}=0.005)$. As a result, preventing symptomatic venous thromboembolism should be associated with real reductions in non-drug costs. Additional savings could also be achieved through further reductions in recurrent symptomatic events and in related long-term events such as post-thrombotic syndrome and chronic thromboembolic pulmonary hypertension. This means that fewer health-care resources may be required. The savings achieved will depend on the health-care system of the different countries in which rivaroxaban will be prescribed. An economic model has been developed to assess the impact of the superior efficacy of rivaroxaban on health-care costs across a range of European countries. One such analysis, conducted from the Italian Healthcare Service perspective, used data from the RECORD1, 2 and 3 studies and included the impact of difference in symptomatic venous thromboembolism and related long-term complications. ${ }^{26}$ This study showed that rivaroxaban might reduce venous thromboembolism substantially, and could potentially generate important non-drug cost savings in this population. ${ }^{26}$

In conclusion, this pooled analysis shows that the efficacy of rivaroxaban $10 \mathrm{mg}$ once daily, commenced six to eight hours following surgery, is superior to that of enoxaparin $40 \mathrm{mg}$ once daily commenced the evening prior to surgery, without an increase in the risk of major bleeding, in patients undergoing elective hip and knee replacement surgery. Furthermore, this improved efficacy was also observed during treatment and the entire follow-up periods.

This study was supported by Bayer Schering Pharma AG and Johnson \& Johnson Pharmaceutical Research \& Development. T-J Bandel, M. Homering and F. Misselwitz are employees of Bayer Schering Pharma AG. All other authors received honoraria as members of the Steering Committees and have served as consultants to Bayer Schering Pharma AG. The authors would like to thank C. Gardner, C. Mongin-Bulewski and R. Dogra for editorial assistance in the preparation of the manuscript, with funding from Bayer Schering Pharma AG.

The author or one or more of the authors have received or will receive benefits for personal or professional use from a commercial party related directly or indirectly to the subject of this article.

\section{References}

1. Geerts WH, Bergqvist D, Pineo GF, et al. Prevention of venous thromboembolism: American College of Chest Physicians evidence-based clinical practice guidelines (8th Edition). Chest 2008;133:381-453.

2. Bergqvist D. Long-term prophylaxis following orthopaedic surgery. Haemostasis 1993;23:27-31.

3. Kearon C. Natural history of venous thromboembolism. Circulation 2003;107:12230.

4. Cohen AT, Agnelli G, Anderson FA, et al. Venous thromboembolism (VTE) in Europe: the number of VTE events and associated morbidity and mortality. Thromb Haemost 2007:98:756-64.

5. Cohen AT, Tapson VF, Bergmann JF, et al. Venous thromboembolism risk and prophylaxis in the acute hospital care setting (ENDORSE study): a multinational cross-sectional study. Lancet 2008;371:387-94.

6. Friedman RJ, Gallus AS, Cushner FD, Fitzgerald G, Anderson FA Jr. Physician compliance with guidelines for deep-vein thrombosis prevention in total hip and knee arthroplasty. Curr Med Res Opin 2008;24:87-97. 
7. Perzborn E, Strassburger J, Wilmen A, et al. In vitro and in vivo studies of the novel antithrombotic agent BAY 59-7939: an oral, direct Factor Xa inhibitor. J Thromb Haemost 2005:3:514-21.

8. Eriksson BI, Borris LC, Friedman RJ, et al. Rivaroxaban versus enoxaparin for thromboprophylaxis after hip arthroplasty. N Engl J Med 2008;358:2765-75.

9. Kakkar AK, Brenner B, Dahl OE, et al. Extended duration rivaroxaban versus shortterm enoxaparin for the prevention of venous thromboembolism after total hip arthroplasty: a double-blind, randomised controlled trial. Lancet 2008;372:31-9.

10. Lassen MR, Ageno W, Borris LC, et al. Rivaroxaban versus enoxaparin for thromboprophylaxis after total knee arthroplasty. N Eng/ J Med 2008;358:2776-86.

11. No authors listed. International conference on harmonisation of technical requirements for registration of pharmaceuticals for human use. Guideline for good clinical practice. http://www.ich.org/LOB/media/MEDIA482.pdf (date last accsesed 6 April 2009).

12. No authors listed. World Medical Assocation Declaration of Helsinki. Ethical principles for medical research involving human subjects. http://www.wma.net/e/policy/ pdf/17c.pdf (date last accessed 6 April 2009).

13. Rabinov K, Paulin S. Roentgen diagnosis of venous thrombosis in the leg. Arch Surg 1972;104:134-44.

14. No authors listed. Medical Dictionary for Regulatory Activities. http://www.meddramsso.com/MSSOWeb/index.htm (date last accessed 6 April 2009).

15. Warwick D, Friedman RJ, Agnelli G, et al. Insufficient duration of venous thromboembolism prophylaxis after total hip or knee replacement when compared with the time course of thromboembolic events: findings from the Global Orthopaedic Registry. J Bone Joint Surg [Br] 2007;89-B:799-807.

16. Cook TM, Mihai R, Wildsmith JA. A national census of central neuraxial block in the UK: results of the snapshot phase of the Third National Audit Project of the Royal College of Anaesthetists. Anaesthesia 2008;63:143-6.
17. Rosencher N, Bonnet MP, Sessler DI. Selected new antithrombotic agents and neuraxial anaesthesia for major orthopaedic surgery: management strategies. Anaesthesia 2007:62:1154-60.

18. Xarelto® Summary of Product Characteristics. http://www.xarelto com/html/ downlads/Xarelto_Summary_of_Product_Characteristics_30Sept2008.pdf (date last accessed 31 March 2009)

19. Gan TJ. Risk factors for postoperative nausea and vomiting. Anesth Analg 2006;102:1884-98.

20. Iorio A, Guercini F, Pini M. Low-molecular-weight heparin for the long-term treat ment of symptomatic venous thromboembolism: meta-analysis of the randomized comparisons with oral anticoagulants. J Thromb Haemost 2003;1:1906-13.

21. Turpie AG, Bauer KA, Eriksson BI, Lassen MR. Fondaparinux vs enoxaparin fo the prevention of venous thromboembolism in major orthopaedic surgery: a metaanalysis of 4 randomized double-blind studies. Arch Intern Med 2002;162:1833-40.

22. Perzborn E, Harwardt M. Recombinant factor VIla partially reverses the effects of the factor $\mathrm{Xa}$ inhibitor rivaroxaban on thrombin generation, but not the effects of thrombin inhibitors, in vitro. J Thromb Haemost 2007;5 (Suppl 2):Abstract.

23. Perzborn E, Tinel H. FEIBA reverses the effects of a high dose of rivaroxaban in rats. Pathophysiol Haemost Thromb 2008;36(Suppl 1):A40.

24. Tinel H, Huetter J, Perzborn E. Recombinant factor Vlla partially reverses the anticoagulant effect of high-dose rivaroxaban: a novel, oral factor Xa inhibitor in rats. $J$ Thromb Haemost 2007;5 (Suppl 2):Abstract.

25. Bullano MF, Willey V, Hauch 0, et al. Longitudinal evaluation of health plan cost per venous thromboembolism or bleed event in patients with a prior venous thromboembolism event during hospitalization. J Manag Care Pharm 2005;11:663-73.

26. Negrini C, Diamantopoulos A, Forster F, et al. Prophylaxis with rivaroxaban against venous thromboembolism (VTE): a cost-consequence analysis from the perspective of the Italian Healthcare Service. Value Health 2008;11:534 